\title{
Padres y madres ante las tareas escolares: La visión del profesorado
}

\author{
Lara Fernández-Freire Álvarez $\left(^{*}\right)$, Beatriz Rodríguez-Ruiz ${ }^{* *}$ y Raquel-Amaya Martínez-González ${ }^{* *}$ ) \\ ${ }^{*}$ ) Colegio Público Alfonso Camín (Asturias) - España (**) Universidad de Oviedo - España
}

\begin{abstract}
RESUMEN
En este estudio se analiza la percepción que tiene el profesorado de centros públicos de Educación Infantil y de Educación Primaria acerca de la implicación de los padres y de las madres en el proceso educativo de sus hijos e hijas y en sus tareas escolares. Se estudian también las posibles dificultades que tienen para ello. La muestra está configurada por 78 docentes de 30 centros ubicados tanto en zonas urbanas como rurales del Principado de Asturias (norte de España). La recogida de información se ha realizado con la Escala de Relaciones entre el Centro Escolar y la Familia, de Martínez-González (1994), obteniendo una fiabilidad adecuada con el índice Alfa de Cronbach. Se han efectuado análisis descriptivos y comparativos de dos grupos con la $t$ de Student teniendo en cuenta el género de los progenitores. Los resultados indican que el profesorado percibe una adecuada implicación de ambos padres en el proceso de aprendizaje académico de sus hijos e hijas en estas etapas escolares iniciales. No obstante, indican que las madres se involucran más y hacen frente con más frecuencia a dificultades derivadas de la conciliación entre la vida familiar, laboral y personal. El estudio concluye con algunas orientaciones educativas y con la propuesta de nuevas líneas de investigación, entre las que se incluye contar con información procedente de los propios padres y madres.
\end{abstract}

Palabras Clave: Tareas escolares, profesorado, familia, Educación infantil, Educación primaria.

\section{Parents and homework. The teachers' perspective}

\section{ABSTRACT}

This study examines the perception of early childhood and primary education teachers in state funded schools as regards the involvement of fathers and mothers in their children's learning and their schoolwork. The study also examines the possible difficulties that parents face to do this. The sample is composed of 78 teachers of 30 centres located both in urban and rural areas of the Principality of Asturias (North of Spain). To gather information, the study applied the School-Family Relationship Scale, by Martínez-González (1994), obtaining an adequate reliability Cronbach\&\#39;s Alpha index. Descriptive and comparative analysis of two groups were conducted with Student's t taking into account the gender of the parents. The results indicate that teachers perceived an adequate involvement of both parents in their children\&\#39;s academic tasks and process in these early stages of school. However, these results suggest that mothers engage more and deal more frequently with difficulties arising from the conciliation between family, work and personal life. The study concludes with some educational guidelines and proposal of new lines of research, including gathering information from parents and mothers.

Keywords: Homework, teacher's perceptions, father, mother, Primary Education.

\section{Introducción}

Las tareas escolares se debaten de manera constante en el ámbito educativo (Cooper, 2001; Cooper y Valentine, 2001; Deslandes, 2009; Pan et al., 2013). En los últimos años se ha incrementado el cuestionamiento social sobre su necesidad (Bailén y Polo, 2016; Consejo Escolar de la Comunidad de Madrid, 2017; Ministerio de Educación de Ecuador, 2016; Sánchez, 2016). En España, el Instituto Nacional de Evaluación Educativa (INEE, 2014) las entiende como una oportunidad de aprendizaje y de fortalecimiento educativo, a diferencia de otras posturas que las consideran una carga para el alumnado y para las familias (OECD, 2016). Los resultados del Informe PISA de 2014 y 2015 (OECD, 2016), en el que participaron 72 países, indican que las tareas escolares guardan una relación positiva con el rendimiento académico (Bailén y Polo, 2016; INEE, 2016), al contribuir a afianzar conceptos, reforzar y aplicar conocimientos y afrontar posibles dificultades en su realización. En España, el Consejo Escolar de la Comunidad de Madrid (2017) indica que hacer "deberes" tiene un impacto positivo en el rendimiento del alumnado, observándose siete puntos porcentuales de diferencia entre el alumnado que los hace $(88 \%)$ y quienes no los hacen.

La complejidad del tema requiere evitar una respuesta única sobre la pertinencia o no de estas tareas escolares, y evidencia 
la necesidad de seguir profundizando en su análisis (Pan et al.. 2013), en especial, desde la perspectiva del profesorado de las primeras etapas educativas. De acuerdo con Cooper (1989), citado en Pan et al. (2013), la expresión tareas escolares se entiende como “aquellas tareas asignadas por los maestros a los estudiantes para realizarlas fuera del horario escolar, con el fin de que las habilidades y conocimientos adquiridos puedan transferirse a otros ambientes" (p.13). En este sentido, se entienden las tareas escolares como actividades que complementan los conocimientos adquiridos en el aula, y que la mayoría de las veces serán compartidas con la familia por el alumnado más joven (Murillo y Martínez-Garrido, 2013). Se trata, por tanto, de un proceso de aprendizaje formal que se da en un contexto no formal -el contexto familiar-, planteándose la necesidad de la implicación parental en el proceso de aprendizaje de los hijos e hijas desde el inicio de la escolaridad (García, 2016). Esta implicación se espera también al considerar las actuales metodologías activas desarrolladas en los centros escolares, como el aprendizaje-servicio, las comunidades de aprendizaje, o el aprendizaje basado en proyectos colaborativos (Maldonado, 2008), que dotan al alumnado desde las primeras etapas escolares, de protagonismo en la construcción de sus conocimientos, dentro y fuera del aula, como parte de su desarrollo integral, interrelacionando los diferentes contextos de socialización en su proceso de aprendizaje (Bronfenbrenner, 1987). En estas metodologías, el profesorado trabaja una temática más allá de las aulas, pidiendo al alumnado que lea, investigue y trabaje también en casa (Kohn, 2013).

\section{Tareas escolares y rendimiento escolar}

De acuerdo con la Association of California School Administrators (2007) para que las tareas escolares en casa sean eficaces y repercutan significativamente en el rendimiento académico han de estar conectadas con los objetivos de aprendizaje de cada etapa educativa y plantear un volumen de trabajo asumible por el alumnado. Además, deben apoyar las metas educativas del centro, tener en cuenta la diversidad del alumnado, y fortalecer el vínculo entre el centro escolar y las familias (Deslandes y Cloutier, 2005; Protheroe, 2009). De darse estas condiciones, la mayoría de los estudios realizados concluyen que "los deberes" repercuten positivamente en el rendimiento académico (Torres, 2014). No obstante, algunos autores como Kohn (2013, citado por el Consejo Escolar de la Comunidad de Madrid, 2017), consideran que no hay resultados claros que demuestren esta relación. Por su parte, Cooper (1989), tras revisar más de 120 estudios en los que se incluían tanto aquellos que indicaban efectos positivos, como los que aportaban resultados difusos, concluyó que el aprovechamiento de las tareas escolares por parte del alumnado y su repercusión positiva en el rendimiento académico, incrementa a medida que se avanza de curso y etapa escolar; idea que también apuntan Núñez et al. (2012).

Suponiendo que los efectos positivos existen, es necesario considerar el grado de dedicación necesaria del alumnado a estas tareas. Bailén y Polo (2016), refiriéndose al informe PISA 2009, plantean un máximo de cuatro horas diarias en educación secundaria, ya que el tiempo adicional tiene un impacto no significativo. En contraposición con estos datos, el informe de la OECD (2013) muestra que en España el tiempo dedicado a las tareas escolares supera en 1,7 horas las cuatro diarias recomendadas. Esta idea es también recogida por el Australian Institute of Family Studies (Edwards, 2012), que constata que la carga diaria de deberes del alumnado español es similar a la carga semanal de la mayoría (59\%) del alumnado australiano de 10-11 años; un fenómeno que empieza cada vez a edades más tempranas, afectando al alumnado de los primeros cursos de Educación Primaria e incluso de Educación Infantil (Consejo Escolar de Navarra, 2012).

Kohn (2013) recoge que, entre 1981 y 2002, el incremento de las tareas escolares habría pasado del $36 \%$ al $64 \%$, principalmente en la primera etapa de la Educación Primaria, e incluso algo de la Educación Infantil (entre los 5-9 años); lo que implica que el alumnado dedica amplios periodos de tiempo a estas tareas en el hogar, con la necesidad de la correspondiente supervisión e implicación familiar; situación que puede dificultar desarrollar otras actividades fuera del horario escolar en el ámbito familiar (Bailén y Polo, 2016). En este sentido, el Ministerio de Educación de Ecuador (2016) sugiere que las tareas escolares en casa en edades tempranas consistan en leer, jugar, hablar e interactuar con los niños y niñas, y dejar para los últimos cursos de la Educación Primaria y para la Educación Secundaria aquellas que requieran una mayor implicación y saber hacer autónomo por parte del alumnado.

\section{Las familias ante las tareas escolares}

De acuerdo con Núñez et al. (2005), el seguimiento de las tareas escolares por parte de la familia es de gran ayuda para la autorregulación y la creación de rutinas y hábitos de estudio en el alumnado. En la investigación realizada por el Consejo Escolar de Navarra (2012), se obtuvo que el 90\% de las familias se implicaba de una u otra forma, y el 70\% controlaba diariamente estas actividades. Los resultados de Pérez-Díaz, Rodríguez y Sánchez (2001) coinciden con los anteriores, indicando que normalmente el alumnado recibe ayuda de algún familiar: el $43 \%$ de las familias consideran que casi siempre o siempre ayudan a su hijos e hijas, y el 14\% que los ayudan bastantes veces; estos porcentajes disminuyen en Educación Secundaria a un 9\%, observando que la implicación familiar se reduce conforme aumenta la edad.

En la Educación Infantil y en la Educación Primaria, los datos indican que dos tercios de este alumnado reciben ayuda de su familia; Martín, Rodríguez y Marchesi (2005) apuntan que un 79,6\% de las familias consideran que colaboran en las tareas escolares de sus hijos e hijas, y Madrona (2001) observa que son las familias del alumnado más pequeño quienes revisan más las tareas diarias $(81,2 \%)$; el porcentaje desciende sustancialmente cuando terminan la Educación Primaria (37\%). Así pues, las familias consideran que están implicadas en el proceso educativo de sus hijos e hijas, destacando algunos indicadores de comportamiento como animarles a que se esfuercen, ofrecerles un lugar tranquilo para estudiar, propiciar que desarrollen hábitos de alimentación y descanso apropiados, compartir tiempo para comentar lo que ha ocurrido durante el día en el centro, participar en las actividades que organiza el centro, ayudarles con sus tareas escolares (Balli, Demo y Wedman, 1998; Deslandes y Bertrand, 2004; INEE, 2016), etc.; pero también están preocupadas por la cantidad de tareas escolares que llevan sus hijos a casa, como indica la Confederación Española de Asociaciones de Padres y Madres del Alumnado (Consejo Escolar de la Comunidad de Madrid, 2017), señalando la presión a la que se ven sometidos los niños y niñas por la sobrecarga de tareas, ocupando un tiempo cada vez mayor, no sólo tras la jornada escolar, sino también los fines de semana y las vacaciones (Consejo Escolar de Navarra, 2012).

Un aspecto más a considerar es cómo es esta implicación parental en función del género de los progenitores. Fúnez (2014) plantea que son las madres quienes ayudan más a sus hijos e hijas, aunque dispongan de menos tiempo que los padres; esta tendencia se observa en diferentes investigaciones (Gonida y Cortina, 2014; Madrona, 2001; Martínez-González, Rodríguez-Ruiz y Rodrigo, 2012; Pizarro, Santana y Vial, 2013; Valdés, Martín y Sánchez, 2009). El estudio realizado por Pérez-Díaz et al. (2001) 
con 400 familias de alumnado de Educación Primaria muestra que en un $82 \%$ de los casos, es la madre quien más se ocupa, destacando, además, que es ella quien más se involucra cuando las actividades son arduas y complejas (Pizarro et al., 2013).

\section{La perspectiva del profesorado sobre la implicación familiar}

Las investigaciones realizadas al respecto indican que los docentes tienden a percibir falta de apoyo por parte de las familias. En el estudio realizado por Cooper, Robinson y Patall (2006), el $44,2 \%$ del profesorado participante estaba de acuerdo en que la familia se desentiende de la educación de sus hijos e hijas; sólo un $24,2 \%$ discrepaban de esta valoración. El 46,3\% percibían que los progenitores no prestan suficiente atención a las actividades escolares de sus hijos e hijas, elevándose este porcentaje más en Educación Secundaria, según Martín et al. (2005). Esto puede deberse, entre otras cosas, a falta de tiempo (Consejo Escolar del Estado, 2015), de capacitación para ayudar (Cooper et al., 2006) o a una escasa comunicación con los hijos e hijas (Martín et al., 2005). Valdés et al. (2009) sostienen que solo un $47 \%$ de los padres y madres se comunican de forma regular con el profesorado y solo un $35 \%$ tiene conocimiento sobre la actividad del centro, lo que afecta de forma indirecta a su implicación tanto en las actividades escolares de sus hijos e hijas, como en las actividades del centro. No obstante, otros estudios indican que el profesorado sí considera (83\%) que la familia ayuda al alumnado a preparar en casa las actividades solicitadas (Mandrona, 2001).

Por tanto, dada la relevancia del tema analizado y la diversidad de perspectivas que se pueden adoptar, en esta investigación se pretende analizar cómo percibe el profesorado de Educación Infantil y de Educación Primaria la implicación de las familias en las tareas escolares y las dificultades que pueden encontrar para ello. Se pretende averiguar, además, si esta percepción del profesorado varía en función del género de los progenitores. La hipótesis de partida es que en estas etapas educativas el profesorado sí percibe implicación familiar, con mayor dedicación de la madre que del padre.

\section{Método}

\subsection{Participantes}

En el estudio han participado 78 docentes de Educación Infantil (33\%) y Primaria (67\%) de 30 centros educativos públicos de zonas urbanas (61.5\%) y rurales (38.5\%) del Principado de Asturias, a través de un muestreo no probabilístico intencional. Se han seleccionado centros públicos por estimar que incluyen una mayor variabilidad de situaciones familiares y de opciones de participación familiar que los centros concertados y privados (Escardíbul y Villarroya, 2010). Respecto al género, participan sustancialmente más profesoras (81\%) que profesores $(19 \%)$, reflejando la tendencia nacional que señala una mayor presencia de la mujer en Educación Infantil y en Educación Primaria (Sánchez, 2002). Respecto a experiencia docente, predominan quienes tienen hasta diez años de ejercicio profesional (47\%) sobre quienes sobrepasan esos años (34\%).

\subsection{Instrumento y procedimiento}

La recogida de información se realizó con la Escala de Relaciones entre el Centro Escolar y la Familia, en su versión para profesorado, adaptada por Martínez-González (1994) al castellano del original Family-School Relationship Trust Scale, del Partnership for School Success Program (EEUU) (Adams y Christenson,
2000). La escala de la adaptación española presenta una primera parte de datos socio-demográficos, y una segunda compuesta por 58 ítems que analizan la perspectiva del profesorado sobre la relación entre el centro escolar y la familia, clasificados en dos secciones: 1) en una se valora la frecuencia de contactos mediante una escala Likert de cuatro alternativas (1-Nunca; 2-A veces; 3-Casi siempre y 4-Siempre); 2) en otra se analizan actitudes y percepciones también mediante una escala Likert de cuatro alternativas, que expresa grado de acuerdo con las temáticas formuladas (1-Nada de acuerdo; 2-Poco de acuerdo; 3-Muy de acuerdo y 4-Totalmente de acuerdo).

En este artículo se analizan específicamente diecisiete ítems referidos a tareas escolares, clasificados en dos dimensiones de análisis: 1) Implicación familiar en el seguimiento y supervisión de las tareas académicas de los hijos e hijas (nueve ítems) (ejemplo: "Los padres y madres ayudan a sus hijos e hijas en las actividades de aprendizaje") y 2) Dificultades de los padres y madres para ayudar a sus hijos e hijas en dichas tareas (ocho ítems) (ejemplo: "Los padres y madres no saben cómo ayudar a sus hijos e hijas con las tareas escolares"). Para aplicar el instrumento se contactó con 35 centros escolares públicos de Asturias, tanto de áreas urbanas como rurales, solicitando autorización a los equipos directivos para su aplicación al profesorado. Se obtuvo respuesta positiva del 85\% (30 centros). Posteriormente, a través del correo electrónico o telefónicamente, se pidió al profesorado que cumplimentara el instrumento, que se adaptó para su aplicación on-line (google-doc). La fiabilidad de la información obtenida, calculada con el índice Alfa de Cronbach resultó .75, considerado aceptable según Nunnally (1978) y respetable según DeVellis (2003).

\subsection{Análisis de datos}

El análisis de datos se ha realizado con el programa SPSS.22. Se han calculado estadísticos descriptivos de frecuencias, porcentajes y medidas de tendencia central y variabilidad; asimismo, se analizó si la distribución de los datos en los ítems se ajustaba a la curva normal calculando la asimetría y curtosis, considerando valores $[-1 ;+1]$, respectivamente (Frías, Llobell y García Pérez, 2000). Posteriormente se realizaron análisis comparativos de dos grupos independientes con la t de Student para averiguar si existen diferencias estadísticamente significativas al considerar el género del progenitor en la percepción del profesorado sobre su implicación en las tareas escolares de sus hijos e hijas. En cada análisis se tuvo en cuenta la condición de homogeneidad de las varianzas (prueba de Levene). Cuando las diferencias entre grupos resultaron significativas se calculó el tamaño del efecto con la $d$ de Cohen, interpretando su magnitud de acuerdo con los siguientes criterios (Cohen, 1988): tamaño bajo cuando $0<\mathrm{d}<$ .20 , medio-moderado: $.20<\mathrm{d}<.50$ y alto: $\mathrm{d}>.50$, (Cohen, 1988 ); si bien tenemos en cuenta que incluso un tamaño del efecto pequeño puede tener una significación práctica (Kirk, 1996).

\section{Resultados}

La exposición de resultados se realiza considerando cada una de las dimensiones de análisis especificadas. En ambas, todos los ítems cumplieron la condición de normalidad.

\subsection{Percepción del profesorado sobre la implicación de los pa- dres y las madres en el seguimiento y supervisión de las tareas académicas de sus hijos e hijas}

Los análisis descriptivos realizados sobre cómo percibe el profesorado la implicación tanto del padre como de la madre, 
indican que es positiva y similar en ambos progenitores (ver Tabla 1). Consideran que ambos apoyan siempre o casi siempre el proceso de aprendizaje de sus hijos e hijas en estas etapas escolares iniciales: se preocupan de que tengan hábitos saludables de alimentación, sueño y descanso (percepción sobre el padre: $75,8 \%, n=66 ; M=2,91, D T=, 717$; percepción sobre la madre: $77,6 \%, n=76 ; M=2,92, D T=, 729)$, así como un ambiente adecuado en casa para estudiar (percepción sobre el padre: $75,7 \%, n=$ $70 ; M=2,81, D T=, 621$; percepción sobre la madre: $75 \%, n=76$; $M=2,81, D T=, 620)$. También estiman que los padres y madres: 1) cuentan con información adecuada sobre cómo han de ayudar a sus hijos e hijas a realizar sus actividades de aprendizaje en casa (percepción sobre el padre: $70,6 \%, n=68 ; M=3,08, D T=$ ,823; percepción sobre la madre: $74,3 \%, n=74 ; M=3,04$, DT= ,836); 2) realizan con sus hijos e hijas actividades y experiencias que les ayudan a aprender (percepción sobre el padre: $74 \%, n=$ 73; $M=2,98, D T=, 638$; percepción sobre la madre: 79,2\%, $n=77$; $M=3,02, D T=, 623) ; 3$ ) les ayudan en las actividades académicas (percepción sobre el padre: 74,1\%, $n=58 ; M=2,90, D T=$,640; percepción sobre la madre: $82 \%, n=78 ; M=3,01, D T=, 613)$ y 4) tienen interés en comentar con el profesorado las actividades que se realizan en el aula (percepción sobre el padre: $71,9 \%, n=71 ; M=$ $2,94, D T=, 883$; percepción sobre la madre: $80,2 \%, n=76 ; M=3,07$, $D T=, 828)$. No obstante, también consideran que es conveniente que los padres y madres aprendan métodos o estrategias para ayudar a sus hijos e hijas a realizar sus actividades de aprendizaje en casa (percepción sobre el padre: $82,8 \%, n=71 ; M=3,41$, $D T=$,838; percepción sobre la madre: $84,4 \%, n=77 ; M=3,43$, $D T=, 823$ ), dado que un volumen considerable percibe que nunca o solo a veces los padres y madres enseñan a sus hijos e hijas cómo estudiar (percepción sobre el padre: $69,6 \%, n=48 ; M=2,32$; $D T=, 696$; percepción sobre la madre: $61,8 \%, n=76 ; M=2,43$, $D T=, 679)$ ). En todo caso, un número relevante considera que nunca o solo a veces el tema del estudio provoca problemas frecuentes en la familia (percepción sobre el padre: $77 \%, n=74 ; M=2,08, D T=, 812$; percepción sobre la madre: $73,7 \%, n=76 ; M=2,08, D T=, 862$ ).

Tabla 1.

Percepción del Profesorado sobre la Implicación del Padre y de la Madre en las tareas escolares

Los padres y madres ayudan a sus hijos e hijas en las actividades de aprendizaje

El alumnado tiene hábitos de alimentación, sueño y descanso adecuados para poder estudiar

El alumnado tiene en casa un ambiente adecuado para estudiar

Es conveniente que los padres y las madres aprendan métodos o estrategias para ayudar a sus hijos e hijas a realizar sus actividades de aprendizaje en casa

El tema del estudio provoca problemas frecuentes en las familias del alumnado

Realizan con sus hijos e hijas actividades y experiencias que les ayudan a aprender

Enseñan a sus hijos cómo estudiar

Los padres y las madres cuentan con información adecuada sobre cómo han de ayudar a sus hijos e hijas a realizar sus actividades de aprendizaje en casa

Los padres y las madres tienen interés en comentar con el profesorado aspectos relacionados con las actividades de enseñanza y aprendizaje que se realizan en el aula

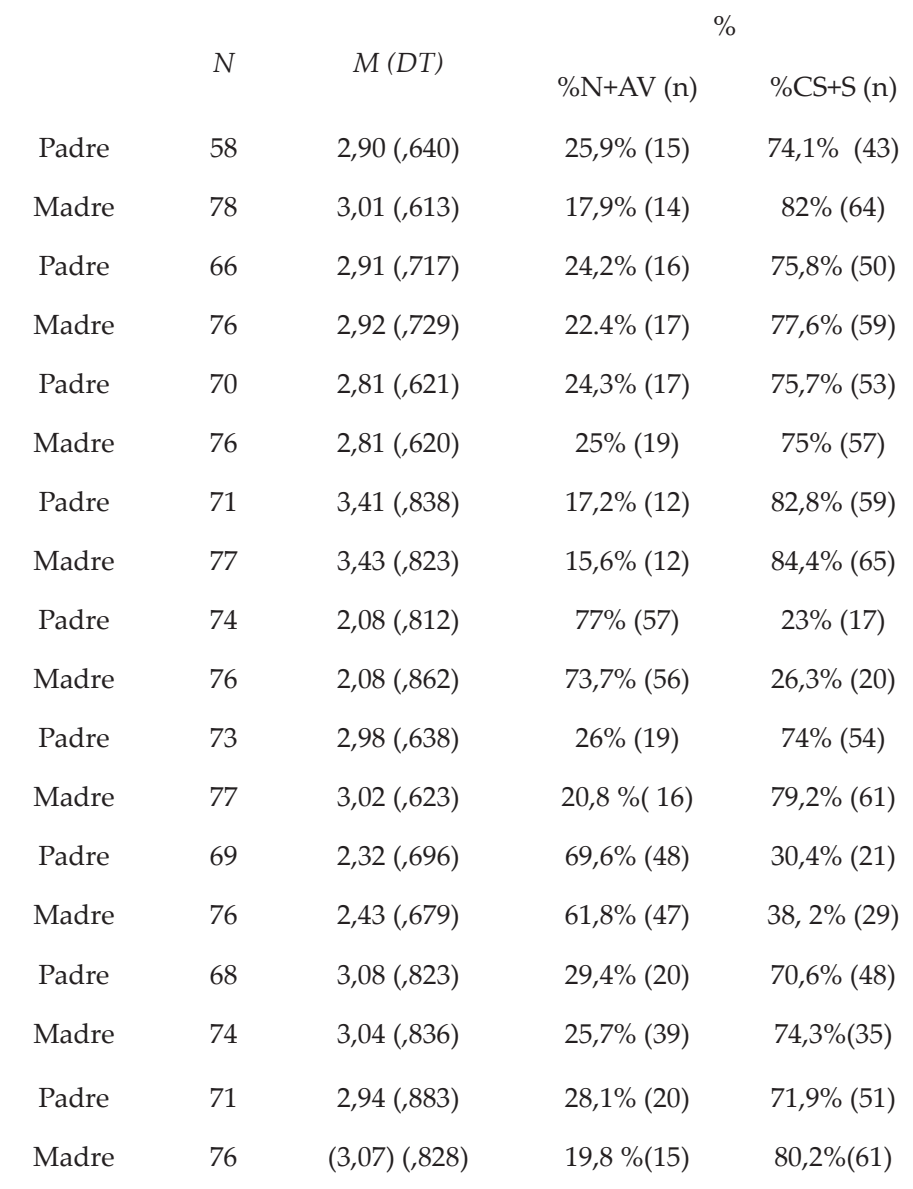

Los análisis comparativos entre grupos tomando en consideración el género del progenitor (ver Tabla 2) indican que el profesorado percibe que las madres se implican significativamente más que los padres en: 1) realizar con sus hijos e hijas actividades que fomentan su aprendizaje, con un tamaño del efecto bajo $\left.\left(t_{72}\right)=-2,977 ; p=, 004 ; d=-, 156\right) ; 2$ ) comentar con el profesorado aspectos relacionados con las actividades escolares de sus hijos e hijas, con un tamaño del efecto bajo $\left(t\left(_{66}\right)=-2,386 ; p=, 020 ; d=-, 127\right)$; $3)$ enseñar a sus hijos e hijas cómo estudiar, con un tamaño del efecto bajo $\left.\left(t{ }_{68}\right)=-2,986 ; p=, 004 ; d=-, 159\right)$ y 4$)$ ayudarles con las actividades de aprendizaje, con un tamaño del efecto bajo $\left(t\left(_{57}\right)=-\right.$ $2,659 ; p=, 010 ; d=-, 206)$. 
Tabla 2.

Diferencias estadísticamente significativas en la percepción del profesorado sobre la implicación del Padre y de la Madre en las tareas escolares y sobre las dificultades que encuentran para ello

$$
\text { N } \quad M(D T) \quad t \quad p \quad d
$$

Percepción del profesorado sobre la Implicación parental en las tareas escolares

\begin{tabular}{|c|c|c|c|c|c|c|}
\hline \multirow{2}{*}{$\begin{array}{l}\text { Los padres y madres ayudan a sus hijos e hijas en las } \\
\text { actividades de aprendizaje }\end{array}$} & Madre & 78 & $3,01(, 613)$ & \multirow{2}{*}{$-2,659$} & \multirow{2}{*}{,010 } & \multirow{2}{*}{,- 206} \\
\hline & Padre & 58 & $2,90(, 640)$ & & & \\
\hline \multirow{2}{*}{$\begin{array}{l}\text { Los padres y madres realizan con sus hijos e hijas } \\
\text { actividades y experiencias que les ayudan a aprender }\end{array}$} & Madre & 77 & $2,98(, 638)$ & \multirow{2}{*}{$-2,977$} & \multirow{2}{*}{,004 } & \multirow{2}{*}{,- 156} \\
\hline & Padre & 73 & $2,92(, 662)$ & & & \\
\hline \multirow{2}{*}{$\begin{array}{l}\text { Los padres y madres enseñan a sus hijos e hijas cómo } \\
\text { estudiar }\end{array}$} & Madre & 76 & $2,43(, 679)$ & \multirow{2}{*}{$-2,986$} & \multirow{2}{*}{,004 } & \multirow{2}{*}{,- 159} \\
\hline & Padre & 69 & $2,32(, 696)$ & & & \\
\hline \multirow{2}{*}{$\begin{array}{l}\text { Los padres y madres tienen interés en comentar con el } \\
\text { profesorado aspectos relacionados con las actividades de } \\
\text { enseñanza y aprendizaje que se realizan en el aula }\end{array}$} & Madre & 76 & $3,07(, 828)$ & \multirow[b]{2}{*}{$-2,386$} & \multirow[b]{2}{*}{,020 } & \multirow[b]{2}{*}{,- 127} \\
\hline & Padre & 71 & $2.93(, 884)$ & & & \\
\hline \multicolumn{7}{|c|}{ Percepción del profesorado sobre las dificultades de implicación parental en las tareas escolares } \\
\hline \multirow{2}{*}{ Los padres y madres tienen que realizar tareas domésticas } & Madre & 74 & $1,89(, 609)$ & \multirow{2}{*}{$-2,416$} & \multirow{2}{*}{,019 } & \multirow{2}{*}{,- 276} \\
\hline & Padre & 60 & $1,73(, 548)$ & & & \\
\hline
\end{tabular}

\subsection{Percepción del profesorado sobre las dificultades de los pa- dres y de las madres para ayudar a sus hijos e hijas en las tareas académicas}

Los análisis descriptivos (ver Tabla 3) indican que, en términos generales, el profesorado que ha colaborado en este estudio no detecta dificultades relevantes ni en los padres ni en las madres para apoyar a sus hijos e hijas de Educación Infantil y de Educación Primaria en su proceso de aprendizaje escolar. No obstante, las escasas dificultades que perciben son diferentes en ambos. En los padres, señalan casi siempre o siempre las tareas domésticas $(68,3 \%, n=60 ; M=1,73, D T=, 548)$ y el hecho de que no necesiten ayudar a sus hijos e hijas porque ya les ayuda otra Tabla 3.

Percepción del Profesorado sobre las Dificultades del Padre y de la Madre para implicarse en las tareas escolares persona $(70,2 \%, n=57 ; M=1,71, D T=, 494)$. Con respecto a las madres, consideran que apenas tienen dificultades y, de haberlas, suelen estar asociadas al cuidado de hijos/as pequeños, personas mayores o enfermas $(15,1 \%, n=73 ; M=1,83, D T=, 728)$, y a que tienen muchas cosas que hacer $(14,7 \%, n=75 ; M=1,93, D T=, 703)$. El profesorado percibe también que en el caso de que las madres no ayuden a sus hijos e hijas, es porque estos no lo necesitan (16\%, $n=75 ; M=1,79, D T=, 740)$. Los análisis comparativos por género solo identifican diferencias estadísticamente significativas vinculadas a dificultades asociadas a realizar tareas domésticas (ver Tabla 2), indicando que el profesorado percibe esta dificultad más en las madres que en los padres, con un tamaño del efecto medio $\left.\left(t{ }_{59}\right)=-2,416 ; p=, 019 ; d=-, 276\right)$.

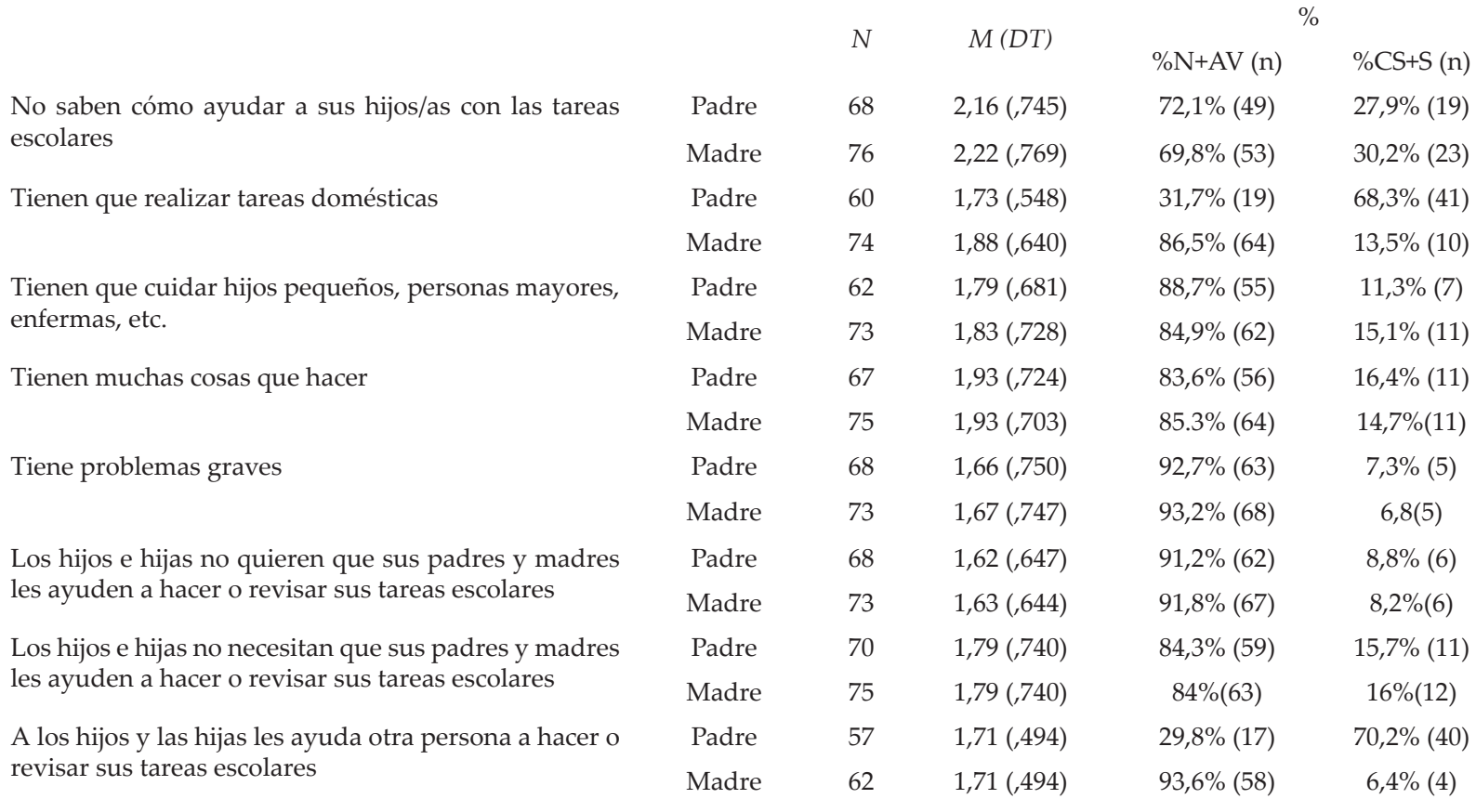




\section{Discusión}

De acuerdo con Balli et al., (1998), el 95\% de los estudiantes rinden mejor en clase cuando reciben ayuda de sus padres y madres. Por ello, el objetivo de este estudio es conocer la percepción que tiene el profesorado de Educación Infantil y de Educación Primaria acerca de la implicación tanto del padre como de la madre en el proceso de aprendizaje académico de sus hijos e hijas, y las posibles dificultades que encuentran para ello. Los resultados obtenidos indican que el profesorado percibe una buena implicación de ambas figuras parentales, proporcionando a sus hijos e hijas experiencias que les ayudan a aprender y apoyándoles con las actividades académicas. De acuerdo con estos resultados, las familias estarían cumpliendo con la expectativa social de colaborar con el centro escolar en la educación de sus hijos e hijas (López, 2012), favoreciendo así una relación mesosistémica entre ambos contextos educativos (Bronfenbrenner, 1987; MartínezGonzález et al., 2012).

En este estudio, además del apoyo en los aspectos directamente académicos, el profesorado ha valorado también positivamente la implicación de las familias en desarrollar en sus hijos e hijas hábitos de alimentación, sueño y descanso, que favorezcan su proceso de aprendizaje (Posada y Taborda, 2012). Esta valoración positiva la realizan tanto con respecto a las madres como a los padres, lo que podría indicar que desde la perspectiva del profesorado de Educación Infantil y de Educación Primaria, las actividades en el hogar respecto a los hijos e hijas son asumidas por ambos progenitores sin mayor dificultad; esto podría entenderse como un indicador social de corresponsabilidad educativa familiar, desdibujando las tradicionales diferencias de género asociadas a la educación de los hijos e hijas, especialmente cuando estos son pequeños y cursan las etapas educativas iniciales.

En lo que respecta a temáticas más académicas, el profesorado que ha participado en este estudio considera que ambos progenitores se preocupan de crear en casa un ambiente adecuado para el aprendizaje fomentado experiencias educativas con sus hijos e hijas; perciben, asimismo, que disponen de estrategias adecuadas para ayudarles en su aprendizaje. Además, consideran que en las etapas escolares de Educación Infantil y de Educación Primaria los estudios de los hijos e hijas no provocan problemas relevantes en el contexto familiar. Tal como sostiene Madrona (2001), estos resultados son indicadores de que los padres y madres apoyan al profesorado en las labores escolares.

El profesorado considera también que los padres y madres se interesan por aprender métodos o estrategias que les permitan ayudar a sus hijos e hijas a estudiar de modo eficaz, y a promover en ellos la autorregulación de sus propias estrategias de aprendizaje (González-Pienda y Núñez, 2005). Estos resultados apoyan la idea de Bailén y Polo (2016), acerca de dotar a las familias de recursos y pautas a seguir para encauzar el proceso de enseñanza-aprendizaje de manera constructiva, limitando las dificultades que puedan tener al respecto. Estas ideas están también en línea con las expuestas por Pan et al. (2013) y Núñez et al. (2012) al considerar que, aunque son los docentes los principales agentes en el proceso de aprendizaje del alumnado, son los propios alumnos y alumnas quienes deben autorregular sus tareas, y en esas primeras edades, los padres y las madres pueden contribuir a ello de modo importante. Por su parte, Cooper (2001) plantea también la necesidad de desarrollar en los estudiantes hábitos y estrategias intelectuales de estudio, ya que a medida que el alumnado progresa en etapas educativas, las familias tienen menos control sobre el contenido académico a trabajar y la implicación parental pierde efectividad. En todo caso, las fami- lias suelen implicarse más en las tareas escolares cuando poseen los conocimientos apropiados para ayudar (Cooper et al., 2006), tal como ha sido percibido por el profesorado que ha participado en esta investigación.

A pesar de que, en líneas generales, en este estudio el profesorado considera que ambos progenitores se implican en las tareas escolares de sus hijos e hijas, si se considera el género, la valoración que reciben las madres tiende a ser mayor que la de los padres. Esta misma apreciación se deriva de las investigaciones realizadas por Valdés et al. (2009), quienes encontraron que las madres dedican más tiempo, con más frecuencia y mayor esfuerzo (Gonida y Cortina, 2014); si bien, no encontraron que el género de los progenitores influyera en los resultados de sus hijos e hijas en las tareas escolares. Fúnez (2014), asimismo, habla de una mayor ayuda de las madres a pesar de ser quienes menos tiempo tienen, dada su dificultad para conciliar la vida familiar y laboral. En nuestro estudio, esta mayor implicación de las madres ha sido percibida por el profesorado también en términos de mostrar mayor interés que los padres por comentar con ellos detalles de las actividades escolares. Este hecho ha sido constatado también por Madrona (2001), quien indica que prácticamente todas las personas que asisten a reuniones con el profesorado son madres o abuelas.

Respecto a las dificultades percibidas por los docentes para que los progenitores ayuden al alumnado con las tareas escolares, identifican pocas, asociándolas a la necesidad de atender a las tareas domésticas, al cuidado de menores o de personas mayores o al volumen de actividades cotidianas a realizar; es decir, son factores externos los que percibe el profesorado que pueden obstaculizar la implicación de ambos progenitores, y no su falta de interés. Estas tareas realizadas más por las madres son percibidas también por los docentes en los estudios realizados por Domínguez (2010) y Martínez-González et al. (2012), quienes añaden que los docentes no solo perciben dificultades en cuanto al cuidado de los hijos o hijas pequeños, sino también de familiares de avanzada edad. En esta línea, Núñez et al. (2012) destacan también la dificultad para conciliar la vida laboral y familiar como un factor que condiciona las actitudes y expectativas de las familias hacia su implicación en las tareas escolares de sus hijos e hijas, que se asocia indirectamente con casos de bajo rendimiento académico. Esta dificultad laboral afecta actualmente a muchas familias y con más frecuencia a las madres que a los padres (Pérez-Díaz et al., 2001; Pizarro et al., 2013), lo que pone de manifiesto la necesidad de fomentar una mejor gestión de la conciliación familiar y laboral (Martínez-González et al., 2012).

Dada esta dificultad, en países como Alemania, Noruega, Grecia o México, se han introducido programas que complementan la jornada escolar y ofrecen una educación suplementaria, como el Programa de Asistencia con los Deberes, de Noruega, o la Alianza Educativa de Alemania (Bailén y Polo, 2016). También en España hay iniciativas similares como el Plan de Orientación, Refuerzo y Apoyo (PROA) promovido desde el curso 2004-2005 por el Ministerio de Educación (2016) o proyectos dirigidos a nivel autonómico, como el Proyecto $11 \times 12$ para la conciliación laboral, familiar y personal iniciado en el Principado de Asturias (2017). A través de esta experiencia, se ha constatado que el profesado manifiesta que las familias colaboran en otros aspectos que facilitan el proceso de aprendizaje escolar, como mantener unos buenos hábitos e higiene del sueño, una dieta equilibrada, con un aporte hídrico distribuido a lo largo del día, así como llevar un estilo de vida saludable, con juegos, ejercicio físico, variedad de estímulos culturales, sociales o artísticos y con un entorno emocional positivo (Consejo Escolar de la Comunidad de Madrid, 2017). 


\section{Conclusión, Limitaciones y Nuevas Líneas de Investigación}

Como conclusión, cabe decir que las investigaciones realizadas sobre la temática analizada (Balli et al. 1998; Cooper, 2001; Cooper et al., 2006; Fernández, 2013; INEE, 2016; López, 2012; Rosario et al., 2009; Núñez et al., 2012) constatan la conveniencia de la implicación parental en las tareas escolares del alumnado en las etapas de Educación Infantil y de Educación Primaria, como factor de promoción escolar, dada su influencia positiva en el proceso educativo y en el aprovechamiento escolar desde las primeras etapas de la escolaridad. El debate se produce cuando la atención se centra en considerar si el profesorado valora solo si las tareas escolares se realizan o no, sin tener en cuenta el contexto social y familiar del alumnado. Según Mourao (2009), la mayoría del profesorado está de acuerdo en que se complementen las actividades del aula con tareas escolares atendiendo a las circunstancias y necesidades sociales y personales del alumnado (Núñez et al., 2005). Los resultados de la presente investigación indican que el profesorado percibe que ambos progenitores apoyan activamente a sus hijos e hijas en sus tareas escolares sin dificultades relevantes en estas etapas. De destacar alguna para ambos progenitores, subrayan la limitación de tiempo y la dificultad para conciliar la vida laboral y familiar; especialmente en el caso de las madres. Este condicionante merece atención social por sus implicaciones para la adecuada dinámica y convivencia familiar, y por los efectos de esta última en los procesos de aprendizaje de los niños y niñas.

Como limitaciones de este estudio y posibles líneas de investigación futura, cabe mencionar el análisis de esta misma temática desde la perspectiva de los propios padres y madres, y de los hijos e hijas, así como la consideración de la etapa de Educación Secundaria, donde los procesos de interacción familiar con los hijos e hijas adolescentes y las demandas escolares se hacen más complejas y exigentes. Cabría también incluir información cualitativa procedente de entrevistas y grupos de discusión para completar y contrastar los datos cuantitativos que aporta este estudio. Por otra parte, la posibilidad de contar con un tamaño de muestra más amplio de profesorado, permitiría generalizar mejor los resultados obtenidos; así como analizar el tema contando también con la participación de centros escolares concertados y privados, donde las dinámicas educativas y las circunstancias socio-demográficas de las familias podrían aportar otros datos diferenciales.

\section{Referencias bibliográficas}

Adams, K. S., \& Christenson, S. L. (2000). Trust and the family-school relationship examination of parent-teacher differences in elementary and secondary grades. Journal of School Psychology, 38(5), 477-497.

Association of California School Administrators (2007). Policy Recommendation for Getting from Facts to Policy: An Education Policy Convening. California: California Education Policy Convening.

Bailén, E., y Polo, I. (2016). Deberes escolares: el reflejo de un sistema educativo. Avances en Supervisión Educativa, 25, 1-36. doi: https://doi.org/10.23824/ase.v0i25.543

Balli, S., Demo, D., \& Wedman, J. (1998). Family involvement with children's homework: An intervention in the middle grades. Family Relations, 47, 149-157.

Bronfenbrenner, U. (1987). La ecología del desarrollo humano. Barcelona: Paidós.

Cohen, J. (1988). Statistical power analysis for the behavioural science. (2nd ed.). Hillsdale, NJ: Lawrence Erlbaum Associates.
Consejo Escolar de la Comunidad de Madrid (2017). Las tareas escolares después de la escuela. Madrid: Consejería de Educación e Investigación.

Consejo Escolar de Navarra (2012). La opinión de los estudiantes sobre las tareas escolares. Pamplona: Junta Superior de Educación.

Consejo Escolar del Estado (2015). Informe 2014 sobre el estado del sistema educativo. Madrid: Ministerio de Educación, Cultura y Deporte.

Cooper, H. (1989). Synthesis of research on homework. Educational Leadership, 47(3), 85- 91.

Cooper, H. (2001). The battle over homework. Thousand Oaks, CA: Corwin Press.

Cooper, H., \& Valentine, J. (2001). Using research to answer practical questions about homework. Educational Psychologist, 36(3), 143-153. doi: https://www.tandfonline.com/doi/ abs/10.1207/S15326985EP3603_1?tab=permissions

Cooper, H., Robinson, J., \& Patall, E. (2006). Does homework improve academic achievement? A synthesis of research, 19872003. Review of Educational Research, 76(1), 1-62. doi: https:// doi.org/10.3102/00346543076001001

Deslandes, R. (Coord.) (2009). International Perspectives on Student Outcomes and Homework: Family-School-Community Partnerships. New York: Routledge.

Deslandes, R., \& Bertrand, R. (2004). Motivation des parents à participer au suivi scolaire de leur enfant au primaire. Revue des Sciences de l'Education, 30(2), 411-434.

Deslandes, R., \& Cloutier, R. (2005). Pratiques parentales et réussite scolaire en fonction de la structure familiale et du genre des adolescents. Revue Française de Pédagogie (INRP), 151, 61-74.

DeVellis, R. F. (2003). Scale development: Theory and applications (2nd Edition). Thousand Oaks, CA: Sage Publications, Inc.

Domínguez, S. (2010). La Educación, cosa de dos: La escuela y la familia. Revista Digital para Profesionales de la Enseñanza, 8, 1-15.

Edwards, B. (2012). Growing up in Australia: The Longitudinal Study of Australian Children: The First Decade of Life. Family Matters, 91, 5-14.

Escardíbul, J. O., \& Villarroya, A. (2010). The inequalities in school choice in Spain in accordance to PISA data. Journal of Education Policy, 24(6), 673-696.

Fernández, C. (2013). Rendimiento escolar y contexto social en educación primaria. Almería: Universidad de Almería.

Frías, M.D, Llobell, J., y García, J.F. (2000). Tamaño del efecto del tratamiento y significación estadística. Psicothema, 12(2), 236-240.

Fúnez, D. (2014). Gestión escolar y la participación de los padres de familia en el proceso educativo de sus hijos. Argentina: Universidad Pedagógica Nacional.

García, R. (2016). Deberes escolares y rendimiento académico. Jaén: Universidad de Jaén.

Gonida, E., \& Cortina, K. (2014). Parental involvement in homework: Relations with parent and student achievement related motivational beliefs and achievement. British Journal of Educational Psychology, 84, 376-396. doi: https://doi. org/10.1111/bjep.12039

González-Pienda, J. A., y Núñez, J. C. (2005). La implicación de los padres y su incidencia en el rendimiento de los hijos. Revista de Psicología y Educación, 1(1), 115-134. doi: 10.15366/reice2016.14.3.007

Instituto Nacional de Evaluación Educativa (INEE) (2014). Sistema estatal de indicadores de la educación. Madrid: Ministerio Español de Educación, Cultura y Deporte.

Instituto Nacional de Evaluación Educativa (INEE) (2016). Alumnos de bajo rendimiento: por qué se quedan atrás y cómo se les puede ayudar. Madrid: Ministerio Español de Educación, Cultura y Deporte. 
Instituto de Desarrollo Académico (Academic Development Institute) (2006). Guía para padres de familia. Illinois: Academic Development Institute.

Kirk, R. E. (1996). Practical significance: A concept whose time has come. Educational \& Psychological Measurement, 56, 746759.

Kohn, A. (2013). El mito de los deberes: ¿Por qué son perjudiciales para el aprendizaje y la convivencia? Madrid: Kaleida.

López, L. C. (2012). La protección constitucional de la familia. Revista de Derechos Sociales, 2(1), 124-131.

Madrona, P. (2001). Actitudes, hábitos y motivaciones de los alumnos ante las tareas escolares en Educación Primaria visto desde la perspectiva de los padres, los maestros y los alumnos. Salamanca: Ediciones Universidad de Salamanca.

Maldonado, M. (2008). Aprendizaje basado en proyectos colaborativos. Una experiencia en educación superior. Laurus, 14(28), 158-180.

Martín, E., Rodríguez, V., y Marchesi, A. (2005). La opinión de los profesores sobre la convivencia en los centros. Madrid: Instituto de Evaluación y Asesoramiento Educativo (IDEA). Centro de Innovación Educativa (CIE-FUHEM).

Martínez-González, R. A. (1994). Diagnóstico de necesidades en la cooperación entre Familia y Centro Escolar. Informe de Investigación. Oviedo: Universidad de Oviedo.

Martínez-González, R. A., Rodríguez-Ruiz, B., \& Rodrigo, Mª . J. (2012). Fathers' and teachers' perception about their partnership. En D. Hiatt-Michael \& H. Z. Ho (Eds.). Promising practices for fathers' involvement in their children's education (pp. 7993). Charlotte, NC: Information Age Publishing.

Ministerio de Educación de Ecuador (2016). Guía de sugerencias de tareas escolares 2016. Madrid: Subsecretaría de Fundamentos Educativos. Dirección Nacional de Currículo.

Mourao, R. (2009). Etapas processuais do trabalho de casa e efeitos auto-regulatórios na aprendizagem do inglês: um estudo com diários de TPC no $2^{\underline{a}}$ ciclo de ensino básico. Braga: Universidade do Minho.

Murillo, F. J., \& Martínez-Garrido, C. (2013). Homework Influence on academic performance. A study of Iberoamerican students of Primary Education. Revista de Psicodidáctica, 18(1), 157- 171. doi: 10.1387/RevPsicodidact.6156

Nord, C. W., Brimhall, D., \& West, J. (1997). Fathers' Involvement in their Children's Schools. NCES 98-091. Washington, DC: U.S. Department of Education, National Center for Education Statistics.

Nunnally, J. C. (1978). Psychometric theory (2nd ed.). New York, NY: McGraw-Hill.
Núñez, J. C., Suárez, N., Fernández, M., Cerezo, R., Rodríguez, C., y Rosario, P. (2012). Tareas para casa, implicación familiar y rendimiento académico. Aula Abierta, 40(1), 73-84.

Núñez, J.C., Rosario, P., Mourao, R., Trigo, J., \& Gonzalez-Pienda, J. (2005). SRL Enhancing Narratives: Testas' (Mis)adventures. Academic Exchange Quarterly, 9(4), 73-77.

OECD (2013), PISA 2012 Results: What Makes Schools Successful? Resources, Policies and Practices (Volume IV). Paris: PISA, OECD Publishing.

OECD (2016). Estudiantes de bajo rendimiento. Por qué se quedan atrás y cómo ayudarles a tener éxito. Paris: PISA, OECD Publishing.

Pan, I., Regueiro, B., Ponte, B., Rodríguez, S., Piñeiro, I., y Valle, A. (2013). Motivación, implicación en los deberes escolares y rendimiento académico. Aula Abierta, 41(3), 13-22.

Pérez-Díaz, V., Rodríguez, J., y Sánchez, L. (2001). La familia española ante la educación de sus hijos. Barcelona: Fundación La Caixa.

Pizarro, P., Santana, A., y Vial, B. (2013). La participación de la familia y su vinculación en los procesos de aprendizaje de los niños y niñas en contextos escolares. Diversitas: Perspectivas en Psicología, 9(2), 271-287.

Posada, D., y Taborda, M. (2012). Reflexiones sobre la pertinencia de las tareas escolares: Acercamientos para futuros estudios. Uni-pluri/versidad, 12(2), 22-33.

Principado de Asturias (2017). Circular de Inicio de Curso 20172018 para centros docentes públicos. Oviedo: Consejería de Educación y Cultura.

Protheroe, N. (2009). Good homework policy=Good teaching. Principal, 89(1), 42-45.

Rosário, P., Mourão, R., Baldaque, M., Nunes, T., Núñez, J.C., González-Pienda, J.A., Cerezo, R., y Valle, A. (2009). Tareas para casa, autorregulación del aprendizaje y rendimiento en matemáticas. Revista de Psicodidáctica, 14(2), 179-192. doi: http://dx.doi.org/10.6018/analesps.31.2.171131

Sánchez, E. (2002). La vocación entre los aspirantes a maestro. Sevilla: Universidad de Sevilla.

Sánchez, M. (2016). Competencias profesionales de futuros profesores de educación infantil al analizar las tareas escolares de simetría. Barcelona: Universidad Autónoma de Barcelona.

Torres, J. (2014). Organización de los contenidos y relevancia cultural. Cuadernos de Pedagogía, 447,50-53.

Valdés, A., Martín, M., y Sánchez, P. (2009). Participación de los padres de alumnos de Educación Primaria en las actividades académicas de sus hijos. Revista Electrónica de Investigación Educativa, 11(1), 1-17. 